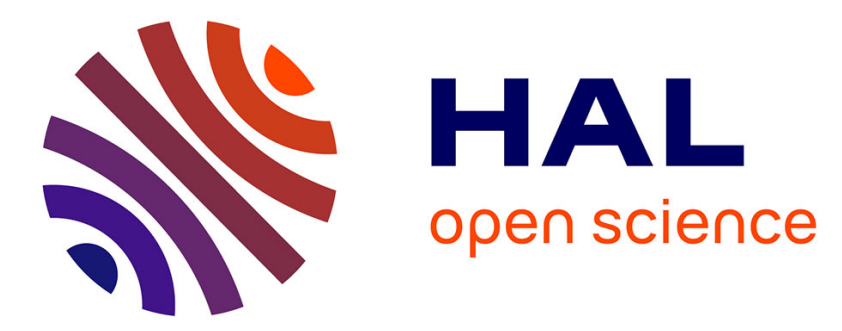

\title{
Rites de groupes et héritages traumatiques à l'adolescence
}

Didier Drieu, Stéphane Corbin

\section{To cite this version:}

Didier Drieu, Stéphane Corbin. Rites de groupes et héritages traumatiques à l'adolescence. Adolescence, 2010, 4 (74), pp.899-906. 10.3917/ado.074.0899 . hal-02480194

\author{
HAL Id: hal-02480194 \\ https://hal.science/hal-02480194
}

Submitted on 15 Feb 2020

HAL is a multi-disciplinary open access archive for the deposit and dissemination of scientific research documents, whether they are published or not. The documents may come from teaching and research institutions in France or abroad, or from public or private research centers.
L'archive ouverte pluridisciplinaire HAL, est destinée au dépôt et à la diffusion de documents scientifiques de niveau recherche, publiés ou non, émanant des établissements d'enseignement et de recherche français ou étrangers, des laboratoires publics ou privés. 


\section{Rites de groupes et héritages traumatiques à l'adolescence}

Les rites à l'adolescence sont souvent appréhendés sous l'angle de la désymbolisation, les conduites à risques chez les adolescents apparaissant alors comme les symptômes d'une déritualisation du cours de la vie et d'une désinstitutionnalisation du lien social. Si cette problématique socio-anthropologique est bien présente, les rites autour de la violence, le risque à l'adolescence mobilisés dans des dynamiques groupales ont aussi une fonction de soutènement des processus de subjectivation. L'enjeu étant de pouvoir s'approprier un héritage souvent traumatique, ces adolescents se retrouvent malheureusement bien souvent entre soi dans des violences fratricidaires.

\section{+ Les conduites à risques : des rites détraqués ?}

Certains adolescents exclus du monde éducatif, vont en effet souffrir de l'absence de repères initiatiques et tenter d'en recréer dans des formes de conduites ordaliques. Les prises de risques peuvent apparaître alors comme des «rituels sauvages, détraqués », la violence renvoyant au dépassement de soi très attendu dans nos mondes modernes, une forme extrême de la fureur de vivre et signant en même temps, un défaut de ritualité ${ }^{1}$ (Baudry, 1991). Ces adolescents manifestent une souffrance tout en remettant en cause un ordre social hors limite qui pousse à l'effacement du corps et de ses frontières. Par rapport au risque, Le Breton

\footnotetext{
${ }^{1}$ La notion de ritualité renvoie tout aussi bien au rituels de l'obsessionnel qu'aux rites plus institutionnalisés. C'est pourquoi nous préférons ici utiliser le terme de rite qui nous paraît mettre en avant davantage le caractère ambigu de ses multiples fonctions
} 
(1996) évoque l'idée d'une recherche de sens par effraction qui peuvent parfois participer à rebouter le symbolique là où la dé-symbolisation est dominante, tenter de maîtriser l'insaisissable chez celui qui manque à trouver sa sécurité de base. Toutefois, l'incidence de la prise de risque sur le jeune est labile et aléatoire, ses effets symboliques sont provisoires car peu étayés par des objets culturels susceptible d'ouvrir sur des processus de transmission. L'intimité du rite et la labilité de ses effets poussent alors ces jeunes à reconduire les conduites à risque, à flirter avec la mort dans ses formes extrêmes jusqu'à l'ordalie.

Lorsque l'indifférenciation menace, quand l'efficacité symbolique de ces rites diminue, la violence des mécanismes émissaires et des conduites sacrificielles peuvent l'emporter. Vecteur possible de désinvestissement, les conduites de prise de risque peuvent se potentialiser dans un ancrage psychopathologique. Plongeant dans l'ennui, dans des groupes involutifs du fait de réseaux inclusifs et restreints, risquant l'oubli, ces adolescents tentent alors de se construire dans des formes d'identité négative avec des conduites d'auto-sabotage, des comportements exacerbés, là où ils ne peuvent se retrouver dans des repères symboliques. Aussi, peut-on parler de plus en plus de rites sauvages dérégulés, de fonctionnements autosensoriels en escalade dans certains groupes où l'adolescent, tout en cherchant la rencontre avec l'altérité, l'inconnu, utilise la logique de l'excès et du défi. Ces conduites se transforment alors en crises qui risquent de s'enkyster dans la croyance en l'expérience de l'extrême et de l'intensité (Ibid). Si ces adolescents sont déjà pris dans des spirales de dépendance avec l'environnement, il y a fort à craindre qu'ils s'enferment progressivement dans une logique addictive par rapport à ces comportements. Les principales figures de délinquance d'autrefois (la transgression, la confrontation à l'autorité) nous apparaissent aujourd'hui plus floues, protéiformes, évoquant plus souvent des conduites de dépendance. P. Givre, en empruntant à la pensée de F. Redl sur la psychologie des bandes, parle de quête 
addictive, une forme d'idéal de performativité comme pouvant être à l'origine des nouvelles conduites délinquantes dans les mégalopoles modernes (Givre, 2002). Il note l'importance, «la pertinence d'interventions éducatives où l'opportunité est donnée à ces jeunes de vivre des expériences de groupe capable de susciter chez eux de nouveaux modes d'investissements (notamment des investissements concernant des objets culturels) ( (Ibid : 114) . Au-delà des investissements susceptible d'étayer le Moi attaqué par la violence du pulsionnel, il importe pour ces adolescents dans ces groupes de reconstituer un appareillage psychique groupal leur permettant de faire travailler les liens et la pulsion dans l'ambiguité du double statut du sujet, individuel et groupal (Kaës, 2007).

\section{+ Rites sauvages et auto-engendrement négatif}

Si la recherche du risque à l'adolescence peut être un moyen de jouer de manière cryptique avec la mort, il peut devenir aussi une manière de_dé-différenciation radicale entre soi, un besoin de convoquer l'altérité là où pointe l'indifférenciation dans les liens familiaux. Ainsi, le désengagement à l'adolescence ne peut qu'échouer face à ces héritages traumatiques. Les adolescents vont alors se retrouver dans des pactes dénégatifs entre pairs (Kaës, 1988).

Si ce fonctionnement en auto-engendrement, un des fantasmes originaires, fait partie des constructions adolescentes, il est ici exacerbé. En effet, il s'agit de s'instaurer dans une forme d'auto-création continuelle de soi-même et de manière autonome à l'image de Protée, le premier homme, pouvant prendre toutes les formes qu'il désire. Dans ce sens, l'autoengendrement est un des mécanismes d'appropriation subjective à l'adolescence, le jeune se portant en avant pour s'approprier son nouveau corps sexué, une pensée personnelle, de nouvelles limites et des idéaux plus matures. Toutefois, ces processus se trouvent contextualisés par les modes de filiation qui vont interférer sur les conditions d'affiliation. En effet, pour transformer ses investissements trop inclus dans la groupabilité interne familiale, 
le jeune doit parvenir à se différencier à partir du groupe social et surtout des pairs. Ces nouveaux liens, groupes pallient à «l'urgence identificatoire» dans laquelle se trouve l'adolescent, l'aidant à construire de nouvelles identifications plus en adéquation avec les aspirations de sa génération (Missenard, 1972). Les doubles, les relations homo-érotiques, puis hétérosexuelles préfigurent en quelque sorte la possibilité d'investir des idéaux plus générationnels et une cellule familiale secondaire.

Le groupe de pairs peut devenir aussi un antidote à l'impossibilité de faire le deuil de l'archaïque fusionnel, à l'incapacité à se dégager des mécanismes régressifs de l'adolescence, poussant le jeune dans un fonctionnement d'auto-engendrement négatif comme dans le fonctionnement des bandes. Ces stratégies même négativantes, font partie du travail de séparation, du dés-assujettissement, d'une lutte contre le désenchantement. Notre époque, en prolongeant considérablement ce temps de l'incertitude sur leurs projets, aurait un pouvoir grossissant sur ces processus d'élaboration et d'internalisation du deuil. Dans un monde aux limites assez floues, marqué par l'individualisme et face au manque d'étayage des objets culturels, les post-adolescents n'auraient «aucun moyen cohérent et collectif de réenchanter leur âme, endeuillée en secret par la perte des objets merveilleux d'autrefois »(Guillaumin, 2001 : 128). Ils doivent donc entrer dans une longue latence et, face au manque de balises culturelles et sociales, utiliser des stratégies multiples et souvent groupales par rapport aux objets. Aussi, ils sont tentés de fragmenter leurs investissements, instrumentaliser leurs rapports avec les autres générations, mettre en suspens indéfiniment leurs choix de vie, rester longtemps instables dans leurs liens d'amitié, dans leurs relations amoureuses, autant de modalités intersubjectives de suspendre le travail de subjectivation. Ils risquent aussi de développer des «conduites traumatophiliques » en rompant brutalement avec les parents ou leurs substituts et fonctionner dans une organisation tribale, indifférenciée 
avec les pairs (Drieu, 2004b). Ces fonctionnements n'ont rien de pathologiques. Leurs buts sont avant tout identitaires et passent par la survie en secret des objets perdus, les objets de l'enfance, les parents idéalisés, relégués à l'état d'objets fétiches, susceptible de préserver l'adolescent des « craintes d'effondrement » (Winnicott, 1963).

Les adolescents absorbés dans des héritages traumatiques ou des modes de filiation narcissique ne peuvent que mobiliser des fonctionnements en auto-engendrement négatif. A plusieurs reprises, nous avons souligné l'importance d'une filiation traumatique liée à des évènements symbolicides dans la famille du jeune suicidant, la négativité à l'œuvre dans la filiation étant susceptible d'être potentialisatrice d'un fonctionnement en auto-engendrement négatif aspirant l'adolescent vers des conduites suicidaires ou para-suicidaires (Drieu, 1999b, 2004a, Drieu, Marty, 2005). Ces mécanismes dans leur dimension paradoxale (autodésengendrement) sont présents dans de multiples troubles et dans les conduites à risque. Ces jeunes paraissent hantés par un originaire traumatique qui les poussent à se confronter dans des comportements en escalade à la vie et à la mort. La violence d'incorporats traumatiques, ces lacunes dans la transmission les poussent vers des conduites de type ordaliques, leurs souhaits de mort leur paraissant plus maîtrisables que l'incertitude de tout autre projet. Au "je ne vous ai pas demandé à naître" si fréquent de l'adolescent(e), le jeune, dans l'escalade, laisse alors entendre violemment que s'il n'a pas choisi de vivre, il peut choisir de mourir.

Ces fonctionnements sont encore plus marqués du sceau de la négativité là où ces adolescents se trouvent confrontés à des «violences transubjectives», des «situations sociales traumatisantes », souvent des traumas multiples qui provoquent chez eux une forme de régression dans l'ambigüité (Amati Sas, 2004). Ces violences sociales continuent à faire son œuvre de mort chez ces adolescents et par résonance dans les institutions (familles, établissements). La violence des secrets provoque une forme de honte chez les divers 
protagonistes. A ces héritages traumatiques, peuvent se rajouter la relégation identitaire : l'exclusion communautaire, sociale, la non reconnaissance d'une histoire spécifique.

Dans ce contexte, le groupe, la bande, de refuge provisoire nous paraît devenir un espace plus clos, potentialisant l'escalade dans les défis mortifères. Les dynamiques d'inclusion négative des bandes de banlieue observées par J. Bordet (1998) se trouvent exacerbées, les adolescents se protégeant alors de menaces identitaires par des formes d'illusion groupale négatives mettant à vif des violences fratricidaires et une escalade dans les défis insensés. A partir d'un travail de régulation auprès d'animateurs-médiateurs dans des centres sociaux, nous avons pu observer le fonctionnement d'un petit groupe d'adolescents, tout particulièrement pendant les révoltes en banlieue parisienne en 2005 .

\section{+ Résonances traumatiques et rites chez des jeunes harkis}

Ces jeunes comme nous l'avons déjà exposé dans un autre écrit ont harcelé les forces de l'ordre pendant deux nuits d'émeutes urbaines qui ont suivi le décès de deux adolescents (Drieu, 2010). Nous évoquons ici brièvement l'histoire de trois jeunes harkis qui ont participé à ces opérations de harcèlement, se faisant appeler les «fellagas », tout en mixant des signes de reconnaissance tant du côté des groupes de soldats harkis que de leurs opposants du FLN. Ces trois jeunes n'ont pas nié les faits qui leur étaient reprochés mais se sont présentés comme des figurants du passé de leurs ancêtres face aux policiers et aux juges. Suivis par plusieurs travailleurs sociaux, ils pourront bénéficier de cet étayage, ce qui semble les avoir contenus dans leur escalade lors de ces journées d'émeutes.

Avant cette révolte, ces jeunes s'étaient organisés dans une bande, plus fantasque que transgressive, s'affirmant dans des signes de reconnaissance, se livrant à des courses poursuites avec une autre bande surnommée les «viets ». Ces jeunes s'étaient redonnés à cette occasion d'autres prénoms plus porteurs de leur origine algérienne en se mettant en jeu 
dans des épreuves initiatiques rappelant fortement l'atmosphère des commandos auxquels appartenaient autrefois leurs père ou grand père.

Dans le cas présent et fort des témoignages des éducateurs et des familles, l'instruction a alors permis d'éclairer le contexte des actes de ces jeunes, en mettant à jour les résonances traumatiques et les dimensions symboliques de leurs conduites. Ainsi, les adolescents ont pu bénéficier de peines alternatives à l'incarcération et entamer grâce au soutien de l'équipe éducative une démarche de soin avec leur famille.

Ces actes, même s'ils ont une fonction opératoire dans l'économie psychique de ces adolescents semblent très en référence avec l'histoire traumatique de leurs ascendants. Confrontés à un originaire traumatique et à un manque de repères d'affiliation (échec scolaire, réseaux sociaux précaires, carence de solidarité familiale, du groupe social), ces adolescents se sont trouvés aspirés dans un fonctionnement en auto-engendrement négatif. Ici, les rites marquant l'appartenance à la bande paraissent avoir maintenu une certaine ambiguïté, les tendances traumatophiliques et les liens à l'environnement semblant pouvoir se jouer sur une scène sociale. En règle générale, les conduites à risque, ces rites sauvages débouchent sur des fonctionnements fratricidaires, les adolescents de ces bandes étant les premiers à devenir l'objet de pratiques sacrificielles comme en témoignent la violence de certains défis, voire d'abus commis dans l'enceinte fermée du groupe. Ici, malgré leur échec scolaire, l'escalade, ces jeunes semblent avoir gardé espoir quant à une possibilité de mettre en scène leur destructivité, à pouvoir fonder des objets plus fiables dans la confrontation à l'autre. Toutefois, en face, les garants de l'autorité, de l'institution (les éducateurs, animateurs-médiateurs, le juge, les familles) ont su également faire la preuve de leur survie lors de ces nuits d'émeutes urbaines.

Certes, les conduites à risque à l'adolescence ne peuvent qu'apparaître bien souvent comme 
une forme détraquée des rites initiatiques, la symbolique du sacrifice dans la transmission n'étant plus aujourd'hui étayée par un environnement institutionnel. Pourtant, certains temps des rituels initiatiques (la phase de marge par exemple) comptent beaucoup, peut être encore plus chez les adolescents vulnérables. S'ils risquent de s'enfermer dans la nasse du groupe, les conduites qu'ils déploient certes dans le défi, dans des jeux cryptés avec l'autorité, ne sont pas sans rappeler l'importance de la dernière phase des cérémonies initiatiques, l'exigence de retrouvaille avec les liens de transmission, la communauté. Ainsi, ces tendances peuvent recouvrir une visée mutative dans la mesure où l'adolescent(e) peut éprouver les capacités nouvelles de l'environnement à faire face à la destructivité. Pour ces adolescents, il s'agit de refonder de nouvelles alliances plus en adéquation avec les idéaux de sa génération mais cette refondation ne peut s'opérer que dans une forme de continuité avec les générations précédentes. C'est pourquoi la figure de l'adulte, les objets culturels, l'institution d'une autorité représentent souvent des balises marquantes pour ces jeunes embarqués dans des conduites quasi-ordaliques.

Drieu Didier Université de Caen, UFR Psychologie Campus 1, Bât. SE, Esplanade de la Paix 14000 Caen -7 rue Bicoquet 14000 Caen tél. 0699583526 E. Mail : didier.drieu@unicaen.fr - Maître de conférences HdR psychologie clinique et pathologie, CERReV, Université de Caen - psychologue, psychothérapeute groupe, CMPP ACSEA, Caen Corbin Stéphane Maître de conférences sociologie, CERReV, Université de Caen

\section{Bibliographie. .}

Amati Sas S. (2004), L'interprétation dans le trans-subjectif : Réflexion sur l'ambigüité et les espaces psychiques, Médecine et hygiène, Psychothérapies, 4, vol. 24 : 207-213

Baudry P.(1991). Corps extrêmes - Approche sociologique des conduites à risque, Paris, L'Harmattan, Coll. Nouvelles études anthropologiques 
Drieu D. (1999b). Les violences automutilatoires à l'adolescence : fonctions et conséquences dans l'approche thérapeutique in Violences à l'adolescence, L'Information psychiatrique, revue mensuelle des psychiatres des hôpitaux, n $^{\circ} 10: 999-1007$

Drieu D. (2004a). Les empreintes traumatiques en jeu dans les tentatives de suicide à l'adolescence, Perspectives Psychiatriques, 43, 2 : 130-136

Drieu D., (2004b). Automutilations, traumatophilie et enjeux transgénérationnels à l'adolescence, Adolescence, 22, $2: 311-323$

Drieu D., Marty F.(2005). Figures de filiation traumatique, Dialogue, 168, 2005 : 5-14

Givre P., (2002). Commentaires du texte de F. Redl «La psychologie des bandes », In Le jeune délinquant, (dir.) F. Marty, Paris, Payot, Coll. Désir : 88-115

Guillaumin J. (2001) Adolescence et désenchantement, Bordeaux, L'esprit du temps, Perspectives psychanalytiques

Kaës R. (1988). Le pacte dénégatif. Eléments pour une métapsychologie des ensembles transsubjectifs, In Figures et modalités du négatif, (dir.) Missenard A. et al., Paris, Dunod

Kaës R.(2007) Un singulier pluriel - La psychanalyse à l'épreuve du groupe, Paris, Dunod, Coll. Psychismes

Le Breton D.(1996), Passions du risque, Paris, Métailié

Le Gall D., Le Van C. (2007), La première fois. Le passage à la sexualité adulte, Paris, Payot. Missenard, A. (1972). Identifications et processus groupal, In : Le travail psychanalytique dans les groupes, ss. la dir. Anzieu D., Kaës R. et al., Paris, Dunod

Winnicott D.-W. (1963). La crainte de l'effondrement, In La crainte de l'effondrement et autres situations cliniques, tr. fr., Paris, Gallimard, NRF, 2000 


\section{Titre : Rites de groupes et héritages traumatiques à l'adolescence}

Résumé : Certaines conduites à risque ont pu être comprises comme symptomatiques d'un « détraquement » des rites à l'adolescence. Ces comportements sont davantage utilisés pour tutoyer la mort, pour réintroduire le jugement ordalique quand des adolescents ne parviennent pas à intégrer l'insaisissable, faute d'être étayés dans leur cheminement par des références à l'institution. Toutefois, ces séquences paradoxales flirtent également, de manière cryptée, avec des mécanismes d'auto-création de soi dans un contexte de filiation traumatique. Ainsi, ces dynamiques reposent à la fois sur le fonctionnement adolescent, de leurs groupes et se trouvent mobilisées en négatif lorsque règnent des violences traumatiques en héritage. Nous proposons de discuter de ces configurations à travers l'exemple d'un groupe de jeunes harkis.

Mots clefs : adolescence, auto-engendrement, dé-ritualisation, groupe, héritage traumatique, rites détraqués, transmission

\section{Title: Group Rites and traumatic inheritances during adolescence.}

Summary: Some high-risk behaviours have been understood as being symptomatic of this "breaking down" of rites during adolescence. Those behaviours are more used to get closer to death, to reintegrate ordalic sentence when teenagers can't integrate the imperceptible, because of a lack of support by institution references during their progression. However, those paradoxical sequences, on a coded way, also flirt with self-creation mechanisms in a context of traumatic filiation. Thus, those dynamics are supported by both teenage process of their groups, and are mobilized on a negative way when traumatic violences heritage reign. We suggest discussing about these configurations with a young harkis group example.

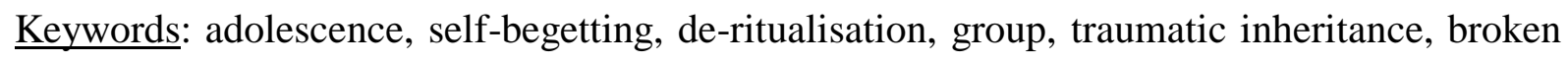
down rituals, transmission. 


\section{Título: Ritos de grupos y herencias traumáticas en la adolescencia.}

Resumen: Ciertas conductas de riesgo pudieron entenderse como sintomáticas de un "desarreglo" de los ritos en la adolescencia. Estos comportamientos se utilizán más para tutear a la muerte, para introducir de nuevo el juicio de prueba cuando adolescentes no consiguen integrar lo inasequible, por falta de apuntalarse en su progreso por referencias a la institución. No obstante, estas secuencias paradójicas flirtean también, de manera codificada, con mecanismos de auto-creación de sí en un contexto de filiación traumática. Así, estas dinámicas se fundan a la vez en el funcionamiento adolescente, de sus grupos y se encuentran movilizadas de modo negativo cuando reinan violencias traumáticas como herencia. Proponemos discutir de estas configuraciones a través del ejemplo de un grupo de jóvenes harkíes.

Palabras claves: adolescencia, auto-engendramiento, de-ritualización, grupo, herencia traumática, ritos desarreglados, transmisión. 